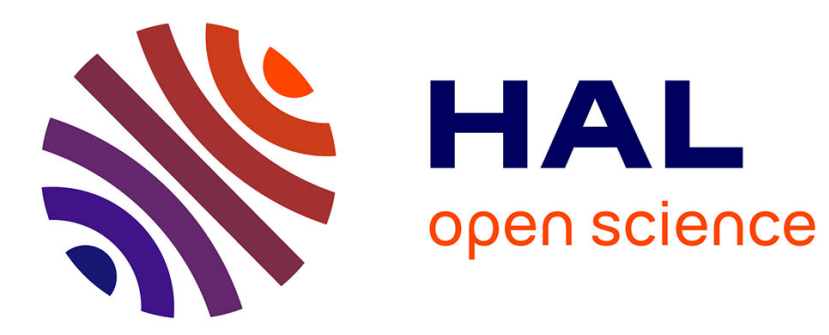

\title{
Velocity Profiles in the Solar Corona from Multi-Instrument Observations
}

Eric Quémerais, Rosine Lallement, Dimitra Koutroumpa, Philippe Lamy

\section{To cite this version:}

Eric Quémerais, Rosine Lallement, Dimitra Koutroumpa, Philippe Lamy. Velocity Profiles in the Solar Corona from Multi-Instrument Observations. The Astrophysical Journal, 2007, 667 (2), pp.1229-1234. 10.1086/520918. hal-00186348

\section{HAL Id: hal-00186348 \\ https://hal.science/hal-00186348}

Submitted on 18 Jul 2020

HAL is a multi-disciplinary open access archive for the deposit and dissemination of scientific research documents, whether they are published or not. The documents may come from teaching and research institutions in France or abroad, or from public or private research centers.
L'archive ouverte pluridisciplinaire HAL, est destinée au dépôt et à la diffusion de documents scientifiques de niveau recherche, publiés ou non, émanant des établissements d'enseignement et de recherche français ou étrangers, des laboratoires publics ou privés. 


\title{
VELOCITY PROFILES IN THE SOLAR CORONA FROM MULTI-INSTRUMENT OBSERVATIONS
}

\author{
E. QuÉmerais, R. Lallement, and D. Koutroumpa \\ Service d'Aéronomie, Verrières le Buisson, France \\ AND \\ P. LAMY \\ Laboratoire d'Astrophysique de Marseille, Marseille, France \\ Received 2007 March 23; accepted 2007 June 3
}

\begin{abstract}
We present a method to derive outflow velocities in the solar corona using different data sets, including solar wind mass flux coming from the SWAN $\mathrm{SOHO}$ instrument, electron density values from LASCO-C2, and interplanetary solar wind velocities derived from ground-based interplanetary scintillation observations (IPS). In a first step, we combine the LASCO electron densities at $6 R_{\odot}$ and the IPS velocities and compare the product to the SWAN mass fluxes. It is found that this product represents the actual mass flux at $6 R_{\odot}$ for the fast wind, but not for the slow wind. In regions dominated by the slow wind, the fluxes derived from SWAN are systematically smaller. This is interpreted as proof that the fast solar wind has reached its terminal velocity at $\sim 6 R_{\odot}$ and expands with constant velocity beyond this distance. On the contrary, the slow solar wind has reached only half of its terminal value and is thus accelerated farther out. In a second step, we combine the LASCO-C2 density profiles and the SWAN flux data to derive velocity profiles in the corona between 2.5 and $6 R_{\odot}$. Such profiles can be used to test models of the acceleration mechanism of the fast solar wind.
\end{abstract}

Subject headings: interplanetary medium — solar wind — Sun: corona

\section{INTRODUCTION}

There is no consensus on the mechanisms of energy deposition and solar wind acceleration in the low corona, especially in the fast wind, although major results have been obtained with the Solar and Heliospheric Observatory ( $\mathrm{SOHO}$ ) instruments. In particular, UV spectroscopy has led to the discovery of high ion temperatures and significantly lower electronic temperatures in coronal holes, as well as strong ion temperature anisotropies (e.g., Kohl et al. 2006; David et al. 1998). A number of models have been developed to reproduce these properties, with damping of high-frequency waves and ion cyclotron heating as favored mechanisms. Because one of the strong difficulties in modeling the solar wind expansion is linked to the heat flux in regions that are not collisional enough for classical transport theory to be valid, kinetic models have also been developed. These models are able to reproduce the acceleration by velocity filtration (e.g., Zouganelis et al. 2005), although they do not reproduce the published temperature anisotropies. Since all different classes of models predict different velocity profiles, accurate measurements of the solar wind flow speed in the acceleration region are required to decide which models are correct.

One of the methods for obtaining the flow speed profile in the acceleration region relies on the measured coronal density profile, as well as the expanding geometry. The velocity profile is simply obtained from the application of the continuity equation, assuming a value for the particle flux coming from the observed region. The flux is inferred from in situ or remote sensing measurements. Munro \& Jackson (1977) pioneered this method using Skylab white-light coronographic images of polar coronal holes. Assuming that the solar flux is roughly constant at all latitudes, they used its average ecliptic value. They concluded that there is a substantial energy addition in the low corona and that the flow accelerates to supersonic speed within $2-3 R_{\odot}$.

Later on, the analysis of solar backscattered Ly $\alpha$ radiation led to the derivation of a significantly lower mass flux in the polar regions (Kumar \& Broadfoot 1979; Lallement et al. 1985). It was shown that the requirement for a nonthermal mechanism is strongly dependent on the density profile and on the out-of-ecliptic mass flux (Lallement et al. 1986). However, our knowledge of the solar wind conditions outside of the ecliptic plane is very limited, as most of the in situ studies of the solar wind are based on data obtained in the ecliptic plane. Because of the small angle $\left(7^{\circ}\right)$ between the ecliptic plane and the solar equatorial plane, this means that most of our knowledge about the solar wind concerns the wind, which originates close to the solar equator.

In this article, we present a new approach to the determination of velocity profiles in the solar corona. The steps are the following:

1. The velocity at large distance from the Sun is obtained from interplanetary scintillation data (Kojima et al. 1998).

2. Using the SWAN interplanetary Ly $\alpha$ data, we can derive the ionization rate of hydrogen at $1 \mathrm{AU}$. After removing the contributions from photoionization and electron impact ionization, we obtain the ionization rate due to charge exchange with the solar wind protons.

3. Using the value of the charge-exchange cross section, which depends on solar wind velocity, we compute the solar wind flux.

4. Assuming radial expansion, we derive the solar wind velocity profile from the values of coronal density and solar wind mass flux. The assumption of radial expansion is not necessary. We could introduce a correction term for nonradial expansion.

5. The previous relation is then applied to the density profiles obtained from the LASCO-C2 polarized white-light images, between 2.5 and $6 R_{\odot}$.

In what follows, we concentrate on a test period from 1996 to 1997 corresponding to a minimum in the solar activity cycle. We briefly present the results of Quémerais et al. (2006) for this period. We then show how mean values of density and velocity can be derived. In the last paragraph, we show how this information 
can be combined with density profiles in the solar corona to give information on velocity profiles.

In our conclusion, we discuss the velocity profiles found in the corona and how they relate to fast-wind or slow-wind conditions. These results are relevant to studies of the mechanism of the solar wind acceleration.

\section{METHOD OF ANALYSIS}

The Ly $\alpha$ background is due to the backscatter of solar Ly $\alpha$ photons by hydrogen atoms present in the interplanetary medium. This UV emission was first identified by instruments on board the OGO-8 spacecraft (Bertaux \& Blamont 1971; Thomas \& Krassa 1971). The hydrogen atoms of the local insterstellar cloud can get very close to the Sun, because of the motion of the solar system relative to the local cloud. In the vicinity of the Sun, the hydrogen atoms are ionized by charge exchange with solar wind protons or photoionized by solar EUV radiation or, with a lesser probability, ionized by collision with hot solar wind electrons.

In the inner heliosphere the spatial pattern of the full-sky Ly $\alpha$ background is carved by the ionizing fluxes from the Sun (Joselyn \& Holzer 1975; Kumar \& Broadfoot 1979). Once a hydrogen atom loses its electron, it cannot backscatter Ly $\alpha$ photons anymore. This creates a hole in the sky pattern. Areas of the sky where ionization is more effective appear dimmer at Ly $\alpha$ because there are fewer hydrogen atoms, which can backscatter solar Ly $\alpha$ photons. The most effective ionization process, roughly 4 times out of 5, is charge exchange with solar wind protons. Even in that case, the hydrogen atom is lost, because the hydrogen atom that captured the electron has the velocity of the solar wind proton, i.e., at least a few hundred $\mathrm{km} \mathrm{s}^{-1}$. The illuminating solar Ly $\alpha$ line is roughly $1 \AA$ wide. In terms of the Doppler shift, this corresponds to $150 \mathrm{~km} \mathrm{~s}^{-1}$. This means that a hydrogen atom with a velocity larger than $150 \mathrm{~km} \mathrm{~s}^{-1}$ is blind to solar Ly $\alpha$ photons, and this results in a loss of hydrogen atoms able to backscatter the solar atoms exactly as in the case of photoionization.

Quémerais et al. (2006) published an analysis of the full-sky background maps obtained with SWAN SOHO between 1996 and 2005. The maps were used to derive the hydrogen ionization rate at $1 \mathrm{AU}$ at all heliographic latitudes. We use these values to derive the solar mass flux and its latitudinal dependence. The ionization rate of hydrogen is the sum of three terms: the photoionization rate, the charge-exchange ionization rate, and the electron impact ionization rate. Photoionization and charge exchange have a radial dependence that varies as the inverse of the square of the distance to the Sun. The photon flux and the solar particle flux have approximately the same radial variation. The electron impact ionization rate also varies with the solar wind electron temperature, which is not constant with distance to the Sun, so its total radial dependence does not vary as the inverse of the square of the distance to the Sun. Using the solar wind electron distribution values obtained by the Wind/Waves experiment (Issautier et al. 2005), we have computed the absolute value of the electron impact ionization rate at 1 AU between 1994 and 2001. We have found values between $10^{-8} \mathrm{~s}^{-1}$ at solar minimum and a few $\times$ $10^{-8} \mathrm{~s}^{-1}$ at solar maximum. The total ionization rate found by Quémerais et al. (2006) varies between $6 \times 10^{-7} \mathrm{~s}^{-1}$ and $1.2 \times$ $10^{-6} \mathrm{~s}^{-1}$, i.e., almost 2 orders of magnitude larger. Then, because the electron impact ionization is small compared to the two other sources of ionization, we neglect it and assume that the total ionization rate of hydrogen varies as the inverse of the square of the distance to the Sun.

The total ionization rate $\beta_{\text {tot }}$ at $1 \mathrm{AU}$ can be written as

$$
\beta_{\mathrm{tot}}=\beta_{\mathrm{exc}}+\beta_{\mathrm{phot}}+\beta_{\mathrm{eii}}=\sigma_{\mathrm{exc}}\left(v_{\mathrm{sw}}\right) v_{\mathrm{sw}} N_{\mathrm{sw}}+\beta_{\mathrm{phot}}+\beta_{\mathrm{eii}} \text {. }
$$

In the previous equation, we neglect the velocity of the hydrogen atom before the solar wind speed $v_{\mathrm{sw}}$. The charge-exchange cross section variation with velocity $\sigma_{\mathrm{exc}}\left(v_{\mathrm{sw}}\right)$ is given by

$$
\sigma_{\mathrm{exc}}\left(v_{\mathrm{sw}}\right) v_{\mathrm{sw}}=\frac{\beta_{\mathrm{tot}}-\left(\beta_{\mathrm{phot}}+\beta_{\mathrm{eii}}\right)}{N_{\mathrm{sw}}} .
$$

Equation (2) can be used to derive the solar wind mass flux from the knowledge of $\beta_{\mathrm{exc}}$ and the density of the solar wind. In the range $0-1200 \mathrm{~km} \mathrm{~s}^{-1}$ the product $\sigma_{\text {exc }}\left(v_{\mathrm{sw}}\right) v_{\mathrm{sw}}$ is a monotonic function of the velocity $v_{\mathrm{sw}}$ and can easily be inverted. The charge-exchange ionization rate is obtained from the SWAN data analysis once a value for photoionization and electron impact ionization has been removed.

The velocity profile $V(R)$ is simply derived using the flux conservation, which can be written as follows, where $R$ is the distance from the Sun center (in $R_{\odot}$ ), and $R_{\odot}$ is $1 \mathrm{AU}$,

$$
N(R) V(R) R^{2}=N_{0} V_{0} R_{0}^{2}
$$

In that case the velocity profile is given by

$$
V(R)=\frac{\beta_{\mathrm{exc}}}{\sigma_{\mathrm{exc}}\left(V_{0}\right)} \frac{R_{0}^{2}}{R^{2}} \frac{1}{N(R)} .
$$

This formalism is used in the following sections.

\section{DATA SETS}

\subsection{Solar Wind Mass Flux: SWAN Data}

The Solar Wind Anisotropy (SWAN) instrument on board the $\mathrm{SOHO}$ spacecraft was developed by a collaboration between Service d'Aéronomie (Verrières le Buisson, France) and the Finnish Meteorological Institute (Helsinki, Finland; Bertaux et al. 1995). Its main purpose is to study anisotropies in the solar wind mass flux and their effect on the interplanetary background (Bertaux et al. 1997). Thanks to the exceptional longevity of the SOHO mission, the data obtained by this instrument almost cover a complete 11 year solar activity cycle.

The most recent analysis of the SWAN data pertaining to the determination of the ionizing fluxes from the Sun was published by Quémerais et al. (2006). In this study, the authors have determined the absolute value of the ionization rate of hydrogen at $1 \mathrm{AU}$ as a function of heliographic latitude and its variation with heliographic latitude. Their analysis covers the period from 1996 to 2005 , i.e., almost a complete solar cycle. The hydrogen ionization rate is related to the solar wind mass flux as shown in equation (2). After correction for solar EUV photoionization and the variation of the charge-exchange cross section with solar wind bulk velocity, the SWAN data give monthly averages of the solar wind mass flux at all latitudes between 1996 and 2005.

\subsection{Solar Wind Density: LASCO Data}

In principle, the solar wind number density at $1 \mathrm{AU}$ can be estimated from in situ measurements performed by numerous spacecraft preeminently restricted to the ecliptic plane. However, results from the Ulysses spacecraft have demonstrated that the density at higher latitudes can be very different from that in the ecliptic (Phillips et al. 1995).

We have therefore relied on the LASCO-C2 white-light images to obtain an estimate of the solar wind density at all latitudes. The LASCO-C2 polarized radiance images $p B$ are routinely generated 

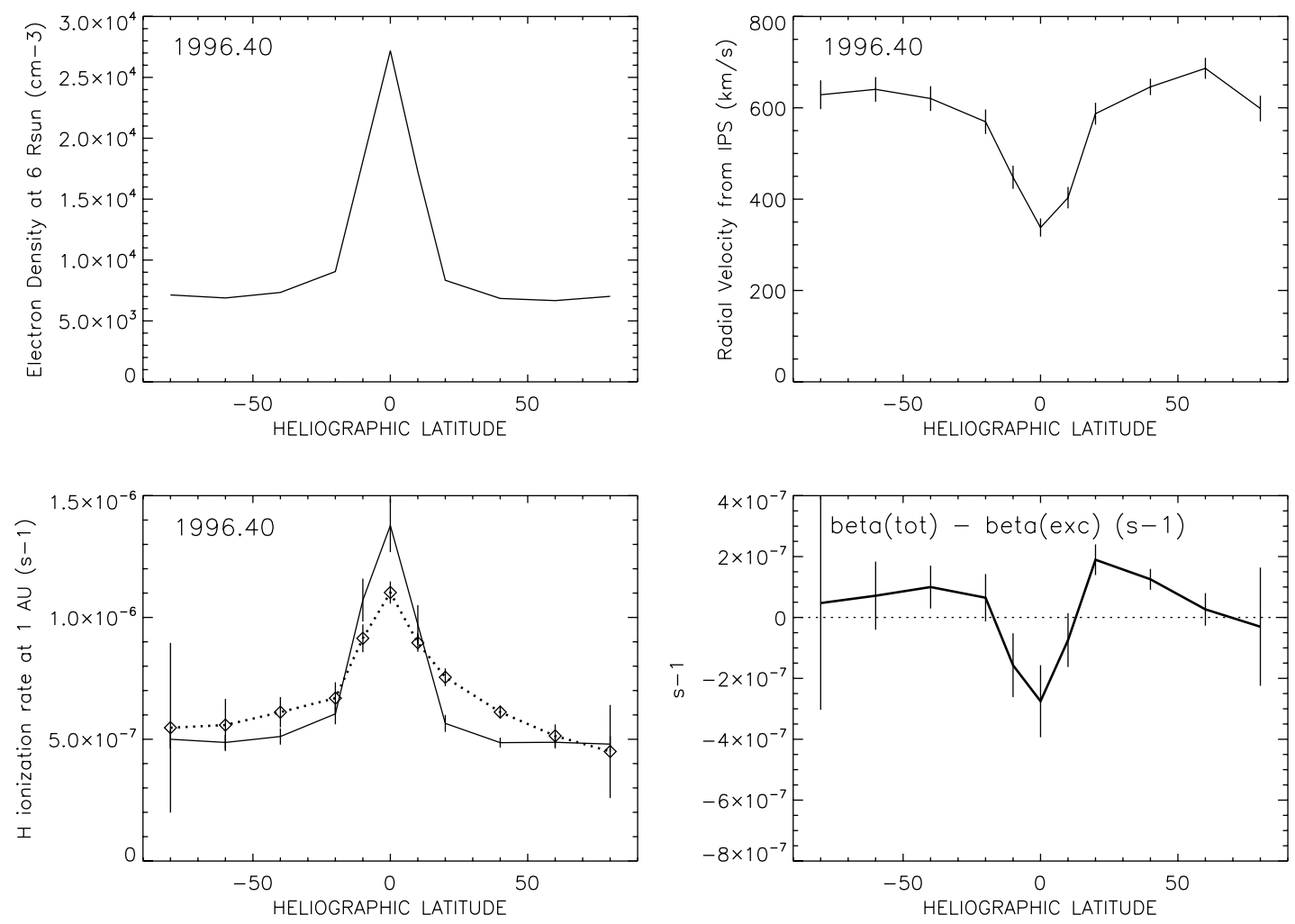

FIG. 1.- Monthly averages of the data used in this analysis. These values were obtained in 1996 March and April. For all panels, the $x$-axis shows the heliographic latitude. Top left: Electron density at $6 R_{\odot}$ obtained from the LASCO-C2 polarized white-light images. The density is strongly enhanced around the equatorial belt. Top right: Average solar wind velocity as deduced from the interplanetary scintillation data. We clearly see the fast wind at high latitudes and the slow wind around the equator. Bottom left: Ionization rate of hydrogen at $1 \mathrm{AU}$. The diamonds joined by a dotted line show the values derived from the SWAN data. The solid line shows the charge-exchange ionization found when using the densities (scaled at $1 \mathrm{AU}$ ) and the velocities displayed in the top panels. Bottom right: Difference between the SWAN values and the values obtained by combining the LASCO-C2 and IPS data. Note the excess produced by the SWAN values at high latitudes, i.e., for the fast solar wind.

from the daily polarization sequences consisting a set of three polarized images taken through the orange filter $(540-640 \mathrm{~nm})$ and polarizers oriented at $60^{\circ}, 0^{\circ}$ and $-60^{\circ}$. Quémerais \& Lamy (2002) have developed a method to invert these images and to produce two-dimensional electron density maps of the solar corona. Compared to our past studies (see also Lamy et al. 1997, 2002; Llebaria et al. 1999), the maps used for these present studies have benefited from refined corrections that successfully remove the instrumental polarization. The values at $6 R_{\odot}$ from the center of the Sun were extracted at all heliographic latitudes. We then computed a monthly average from the daily maps to obtain values with the same sampling as the ionization rate derived from the SWAN data. We discuss below in which cases the density at $6 R_{\odot}$ can be simply extrapolated at $1 \mathrm{AU}$.

\subsection{Solar Wind Terminal Velocity: IPS Data}

As one can see from a quick perusal through the catalog of the OMNIWeb database, various spacecraft have measured the solar wind velocity in the ecliptic plane, but only a few measurements are available at mid and high heliographic latitude. Here we have used the solar wind velocity estimates obtained from interplanetary scintillation data (IPS; Kojima \& Kakinuma 1990). These velocity values are derived from the analysis of scintillations of radio emissions from compact radio sources (Kojima et al. 1998). Because the distribution of radio sources in the sky is not uniform, the sampling of these measurements is not either. These authors have found that the solar wind speed is roughly constant beyond $0.1 \mathrm{AU}$ from the Sun, which means that the acceleration occurs earlier. Here we have averaged the velocity values beyond $0.1 \mathrm{AU}$ from the Sun as a function of the heliographic latitude from which the wind originates. Those averages were made on a monthly basis. It should be noted also that there is a 4 month data gap every year due to weather conditions that prevent ground-based radio observations.

\section{RESULTS}

\subsection{Computation of $\mathrm{H}$ Ionization Rates}

The first step in our analysis is to check whether SWAN results are compatible with the LASCO density values, the IPS velocity estimates, and the assumption that the IPS velocity is reached at the outer edge of LASCO-C2. To do that, we compute the chargeexchange ionization rate at $1 \mathrm{AU} \beta_{\mathrm{exc}}$, where the velocity is derived from the IPS data and the density is equal to the value found from the LASCO images with a radial scaling coefficient. This coefficient expresses the relationship between the density measured by LASCO at $6 R_{\odot}$ and the density at $1 \mathrm{AU}$. For a radial expansion, the product $N V r^{2}$ is conserved. If the acceleration is finished, then the velocity is constant, and the density varies as $1 / r^{2}$.

Figure 1 shows the parameters and calculations obtained in 1996 April and May. The top left panel shows the LASCO-C2 average density at $6 R_{\odot}$ as a function of heliographic latitude. There is a strong enhancement in the equatorial band. The top right panel shows the IPS mean velocity as a function of heliographic latitude for the same period. The bottom left panel shows the ionization rate of hydrogen at $1 \mathrm{AU}$. The diamonds linked by a dotted line correspond to the SWAN data. The solid line corresponds to the value derived from the scaled LASCO density and the IPS velocity data. The computed value represents only ionization through charge exchange. The bottom right panel shows 

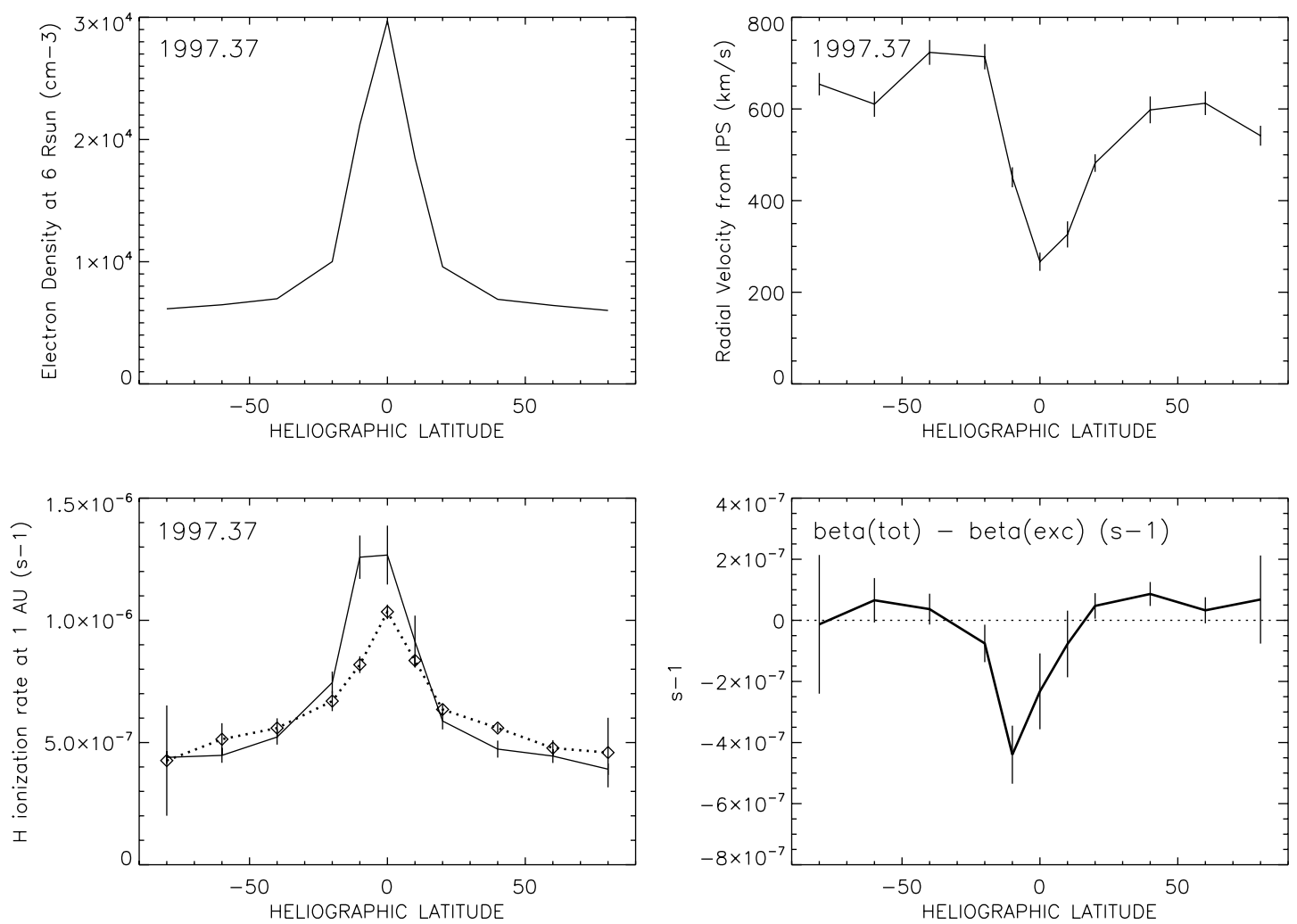

Fig. 2.- Same as Fig. 1, but for data obtained in 1997 Spring. Although the numerical values are not identical, we find the same dichotomy between fast wind and slow wind. As shown in the bottom right panel, the flux deduced from the IPS values and the LASCO-C2 data is too high along the equator. This means that the slow wind has not reached its terminal velocity at $6 R_{\odot}$.

the difference of the two curves shown in the bottom left panel. In this plot, we see two different regimes. In the equatorial band, the computed charge-exchange rate is systematically larger than the SWAN total ionization rate. This means that the velocity between $6 R_{\odot}$ and $1 \mathrm{AU}$ is not constant and that the radially scaled density is overestimated. This also implies that the velocity at $1 \mathrm{AU}$ is larger than the velocity at $6 R_{\odot}$, which means that in the equatorial band the solar wind has not completed its acceleration. Outside of the equatorial band, the SWAN ionization rates are systematically larger than the computed charge-exchange ionization rate. This is consistent with the fact that in this region the solar wind has reached its terminal velocity and that the density simply scales as $1 / r^{2}$ with solar distance. The difference between the two values can be attributed to the sum of photoionization and electron impact ionization.

Figure 2 shows a very similar picture. The values shown here are for 1997 April and May. Once again, the equatorial belt data suggest that the wind is not completely accelerated, while at higher latitudes the ionization rate values agree well.

Figure 3 shows the difference between the SWAN total ionization rate $\beta_{\text {tot }}$ and the charge-exchange ionization rate $\beta_{\text {exc }}$ computed from LASCO and IPS data as a function of the IPS solar wind velocity. All monthly values for different heliographic latitudes have been used. The data are averaged over intervals of $10^{\circ}$ in latitude. Two patterns appear clearly. For velocities larger than $500 \mathrm{~km} \mathrm{~s}^{-1}$, the quantity $\beta_{\text {tot }}-\beta_{\text {exc }}$ is positive. Its mean value is equal to $(5.3 \pm 2.3) \times 10^{-8} \mathrm{~s}^{-1}$. This is consistent with the fact that for the fast-wind conditions (here faster than $500 \mathrm{~km} \mathrm{~s}^{-1}$ ), the acceleration is completed at $6 R_{\odot}$ and that the solar wind density at $1 \mathrm{AU}$ is correctly derived from the LASCO value scaled by $1 / r^{2}$. In that case, the difference between the total ionization rate and the charge-exchange ionization rate is equal to the sum of the hydrogen photoionization rate and the electron impact ionization rate. The value of $(5.3 \pm 2.3) \times 10^{-8} \mathrm{~s}^{-1}$ found here is compatible with previously estimated values. For example, hydrogen photoionization rate estimates for solar minimum conditions range between $5 \times 10^{-8}$ (Rucinski \& Fahr 1991) and $10^{-7} \mathrm{~s}^{-1}$ (Ogawa et al. 1995). We estimated electron impact ionization rates for hydrogen using the Wind/Waves data (Issautier et al. 2005) and found values around $10^{-8} \mathrm{~s}^{-1}$, close to solar minimum.

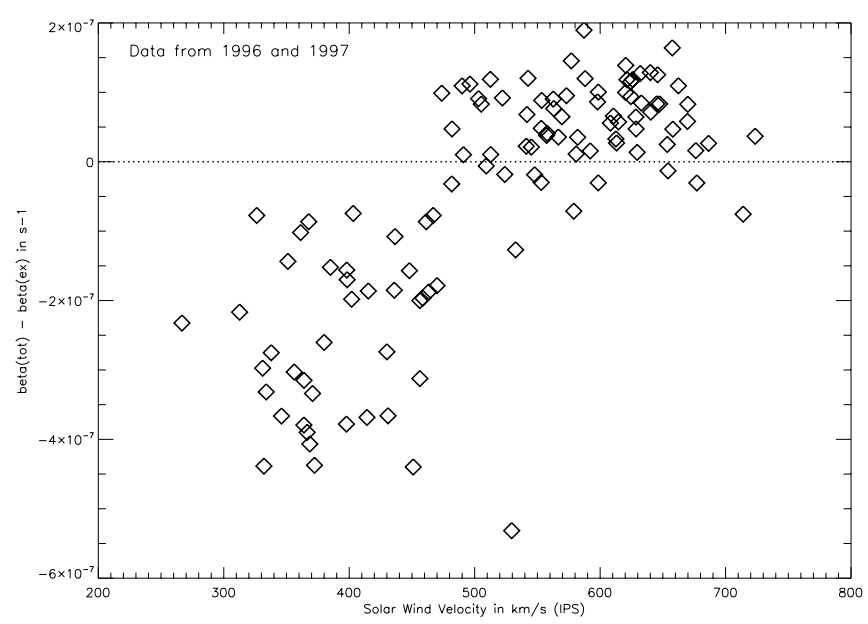

FIG. 3.-Difference between the SWAN total ionization rate at $1 \mathrm{AU}$ and the charge-exchange rate deduced from LASCO density and IPS velocity data. The $x$-axis shows the velocity value from the IPS data. In this plot, we have used all available data from 1996 and 1997 , averaged monthly. The latitude bins are $10^{\circ}$ wide. Here we see the two types of wind, the fast wind $\left(\geq 500 \mathrm{~km} \mathrm{~s}^{-1}\right)$, where the difference is positive, and the slow wind, where it is negative. The average of the fast-wind values gives an estimate of the sum of photoionization and electron impact ionization rates. 


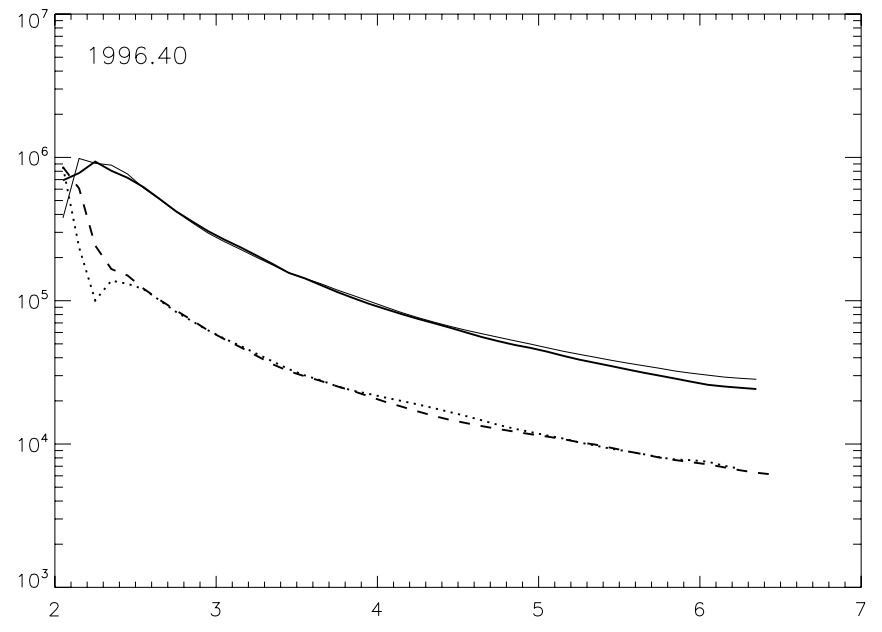

FIG. 4.-Density profiles for 1996 Spring deduced from the LASCO-C2 polarized white-light images. The values correspond to 1 month averages. The top solid lines give the values for the east and west limbs. The two limbs are very similar, which is normal for an average of 1 month of data. The dashed line shows the north pole density profile, and the dotted line shows the south pole profile. There is almost a factor of 10 between polar and equatorial values.

When the IPS velocity value is lower than $500 \mathrm{~km} \mathrm{~s}^{-1}$, we find that the quantity $\beta_{\text {tot }}-\beta_{\text {exc }}$ is negative. As mentioned above, the flux conservation from $r_{i}=6 R_{\odot}$ to $1 \mathrm{AU}$ also contains a ratio $V\left(r_{i}\right) / V(1 \mathrm{AU})$. This suggests that for slow solar wind conditions, the solar wind has not yet reached its terminal velocity and that the ratio $V\left(r_{i}\right) / V(1 \mathrm{AU})$ is smaller than 1 , assuming a radial outflow as defined in equation (3).

Here we find a clear dichotomy between fast solar wind regions at high latitude and slow solar wind regions near the equatorial region.

\subsection{Velocity Profiles in the Corona}

Figure 4 shows the LASCO-C2 electronic density radial profiles in 1996 Spring averaged over the same period as the SWAN data. This results in very regular curves where most transient effects such as coronal mass ejections (CMEs) have been smoothed out. The two solid lines show the east limb and west limb density profiles. The dashed line shows the north pole density profile, and the dotted line shows the south pole density profile. Within $2.5 R_{\odot}$ of the Sun, the data are contaminated by the instrumentscattered light, and the uncertainty is large. The profiles can only be used with confidence between 2.5 and $6 R_{\odot}$. We see that the pole profiles have densities that are 10 times lower than the equatorial values.

Figure 5 displays the velocity profiles derived from the densities in Figure 4 using equation (4). The flux scaling factor $\beta_{\text {exc }} / \sigma_{\text {exc }}\left(V_{0}\right)$ is derived from the SWAN data of the same date. The solid line shows the radial dependence of the solar wind as a function of distance for the average of north and south poles. On the right-hand side of the figure we have plotted the corresponding IPS value around $600 \mathrm{~km} \mathrm{~s}^{-1}$. The dotted line shows the individual profiles derived for the north and south poles. They are very similar to the average profile. From these profiles, we see that the solar wind goes from about $200 \mathrm{~km} \mathrm{~s}^{-1}$ at $2.5 R_{\odot}$ up to $\sim 600 \mathrm{~km} \mathrm{~s}^{-1}$ at $4.5 R_{\odot}$. After a distance of $5 R_{\odot}$, the increase of solar wind velocity is less pronounced, and acceleration is essentially finished at $6 R_{\odot}$.

The dashed line shows the profile derived using the average of the two equatorial density profiles shown in Figure 4. Once again the flux scaling ratio is derived from the SWAN equatorial data

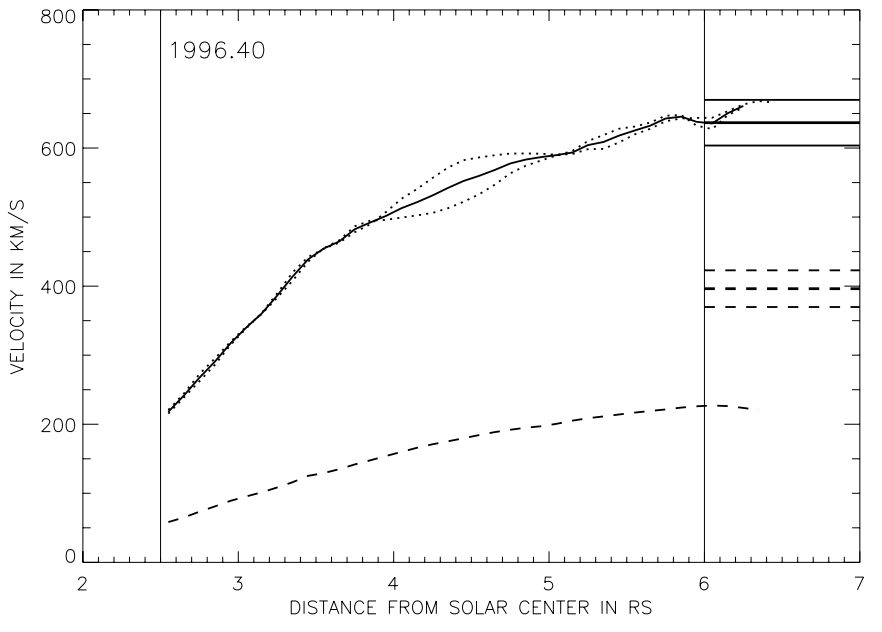

FIG. 5.- Velocity profiles for 1996 Spring deduced from the combination of SWAN, LASCO, and IPS data. The solid line represents the average of the north and south profiles, while the dotted lines show the individual north and south profiles. We see an inflection around 3.5 $R_{\odot}$, which suggests a change in the acceleration at this altitude. The dashed line shows the profile derived for the equatorial solar wind. This profile was derived by assuming a radial expansion and may have to be corrected for nonradial effects. After $6 R_{\odot}$, the lines show the IPS velocity values. For both polar profiles, we see that the profiles reach the terminal value around $6 R_{\odot}$, whereas at the equator the velocity at $6 R_{\odot}$ is only half of its terminal value.

for the same period. The corresponding IPS velocity value is shown on the right-hand part of the plot and has a value close to $400 \mathrm{~km} \mathrm{~s}^{-1}$. We see that for the slow wind, the mean velocity at $6 R_{\odot}$ is only $200 \mathrm{~km} \mathrm{~s}^{-1}$, i.e., half of the terminal velocity. This result is limited by the assumption of radial expansion, which is implicit in equation (4). Although this assumption is quite justified for a polar coronal hole above 5 or $6 R_{\odot}$, this may not be the case for the equatorial slow wind. In that case, a correction term should be applied to take into account the nonradial part of the slow-wind expansion. Furthermore, this profile concerns an average of many transient phenomena that may have had very different individual velocity profiles.

\section{COMPARISON WITH OTHER WORKS}

The present work provides time-averaged velocity profiles at all latitudes. Coronal flow velocities have been obtained in specific regions and at specific times in different ways. The outflow velocity has been inferred from analyses of the Doppler dimming of the intensities of the $\mathrm{O}$ vi $\lambda \lambda 1032,1037$ and H I Ly $\alpha \lambda 1216$ lines, using the UVCS on board SOHO. In particular, Strachan et al. (2000) derived the latitudinal dependence of O5+ outflow velocities between 1.75 and $2.75 R_{\odot}$. Gabriel et al. (2005) derive an outflow velocity of $150-200 \mathrm{~km} \mathrm{~s}^{-1}$ at $2.5 R_{\odot}$ in polar plumes. Kohl et al. (1998), Cranmer et al. (1999), and Antonucci et al. (2000) show that in a polar hole the fast-wind speed reaches $300-400 \mathrm{~km} \mathrm{~s}^{-1}$ near $3 R_{\odot}$. On the other hand, Raymond et al. (1998) use UVCS observations of a Sun-grazing comet at $6.8 R_{\odot}$ and derive an outflow velocity smaller than $640 \mathrm{~km} \mathrm{~s}^{-1}$ in a highspeed wind region. They argued that only $50 \%-75 \%$ of the $1 \mathrm{AU}$ proton energy is reached at $6.8 R_{\odot}$ and that an additional energy deposition is at work beyond this distance.

Above streamers, Strachan et al. (2002) derived a null velocity up to $3.5 R_{\odot}$ and an abrupt acceleration to $90 \mathrm{~km} \mathrm{~s}^{-1}$ at $5 R_{\odot}$ along the streamer axis, while along the streamer legs the velocity has already reached $100 \mathrm{~km} \mathrm{~s}^{-1}$ at $2.33 R_{\odot}$. Based on expanding features, Sheeley et al. (1997) estimated the acceleration of the slow wind above streamers and found it compatible with a 
Parker-type isothermal expansion with values ranging from 150 to $300 \mathrm{~km} \mathrm{~s}^{-1}$ between 5 and $25 R_{\odot}$.

In the open field/fast wind regions, the geometry is extremely simple, and a direct comparison between previous localized measurements and the monthly averaged SWAN/LASCO/IPS results can be performed. Indeed, there is a quite good agreement with SUMER and UVCS results between 2 and $3 R_{\odot}$. The agreement suggests that the fast-wind acceleration is rather homogeneous and constant with time. Note that the flow speed we derived is, however, above the maximum speed at $6.8 R_{\odot}$ deduced from the Sun-grazing comet.

For the slow wind the comparison is not so easy, because it originates in and around closed-field regions, with a more complicated geometry. It has been shown by Strachan et al. (2002) that the profiles vary strongly from the streamer axis to the legs. Since the streamers vary in angle, latitude, and shape, more work is needed to interpret the average profile.

\section{CONCLUSION}

In this article, we have shown how we can retrieve velocity profiles in the corona between 2.5 and $6 R_{\odot}$. This analysis has been applied to data from 1996 and 1997 . We have used monthly averages because the SWAN inversion technique is based on data covering at least one solar rotation. We have found that during solar minimum, there is a strong difference between the velocity profiles derived for the polar and the equatorial regions of the Sun. The polar regions show a fast solar wind with lower density at $1 \mathrm{AU}$, while the equatorial regions are the source of the slow solar wind with increased density. These features were already well known from analysis of the interplanetary scintillation data (Kojima \& Kakinuma 1990) or the Ulysses fast latitudinal scan of 1994 (Phillips et al. 1995).

Using the LASCO density values derived at $6 R_{\odot}$, we have been able to show that the fast wind has a radial expansion with constant velocity beyond $6 R_{\odot}$. This confirms the idea that the fast solar wind is accelerated at low altitudes in the corona and that it has already reached its terminal velocity at $6 R_{\odot}$. This also means that the density ratio between $6 R_{\odot}$ and $1 \mathrm{AU}$ is simply scaled by the ratio of the square of the distance to the Sun [eq. [3], where $V(R)=V_{0}$ and $\left.R=6 R_{\odot}\right]$. As an aside, we can also mention that in this case a combination of the SWAN flux data with the LASCO density values can be used to derive the velocity value at $1 \mathrm{AU}$ without needing the information given by the IPS analysis.

The slow wind originating from equatorial regions of the Sun shows a different behavior. First, we have seen that the density ratio between $6 R_{\odot}$ and $1 \mathrm{AU}$ is not simply equal to the ratio of the square of the distance to the Sun. This is shown by computing the flux using the density and the IPS velocity and comparing to the SWAN value. We see that the SWAN value is always smaller, which means that the velocity ratio between the outer edge of LASCO-C2 and $1 \mathrm{AU}, V\left(6 R_{\odot}\right) / V_{0}$, is smaller than 1.

As shown in Figure 3, the threshold between fast and slow winds is $\sim 500 \mathrm{~km} \mathrm{~s}^{-1}$. Below that threshold, the wind has not reached its terminal velocity at $6 R_{\odot}$, while it has reached its terminal velocity at $6 R_{\odot}$ for values larger than $500 \mathrm{~km} \mathrm{~s}^{-1}$. The velocity profile for the slow wind may be different from the one we have produced if the expansion factor is not purely radial. This may be true at lower altitudes in the corona.

Finally, our velocity profiles provide a test of the models of solar wind acceleration to reveal which mechanisms are really effective. In future developments of this work, we will extend this study to all periods of solar activity. The corresponding SWAN data used to derive the solar wind flux at $1 \mathrm{AU}$ have already been analyzed by Quémerais et al. (2006). This will allow us to study the variation of the acceleration profile with solar activity.

$\mathrm{SOHO}$ is a mission of international cooperation between ESA and NASA. SWAN and LASCO-C2 activities in France are funded by CNES with support from CNRS. SWAN activities in Finland are funded by TEKES and the Finnish Meteorological Institute.

\section{REFERENCES}

Antonucci, E., Dodero, M. A., \& Giordano, S. 2000, Solar Phys., 197, 115

Bertaux, J. L., \& Blamont, J. E. 1971, A\&A, 11, 200

Bertaux, J. L., et al. 1995, Sol. Phys., 162, 403 1997, Sol. Phys., 175, 737

Cranmer, S. R., et al. 1999, ApJ, 511, 481

David, C., Gabriel, A. H., Bely-Dubau, F., Fludra, A., Lemaire, P., \& Wilhelm, K. 1998, A\&A, 336, L90

Gabriel, A. H., Abbo, L., Bely-Dubau, F., Llebaria, A., \& Antonucci, E. 2005, ApJ, 635, L185

Issautier, K., Perche, C., Hoang, S., Lacombe, C., Maksimovic, M., Bougeret, J.-L., \& Salem, C. 2005, Adv. Space Res., 35, 2141

Joselyn, J. A., \& Holzer, T. E. 1975, J. Geophys. Res., 80, 903

Kohl, J. L., Noci, G., Cranmer, S. R., \& Raymond, J. C. 2006, Astron. Astrophys. Rev., 13, 31

Kohl, J. L., et al. 1998, ApJ, 501, L127

Kojima, M., \& Kakinuma, T. 1990, Space Sci. Rev., 53, 173

Kojima, M., Tokumaru, M., Watanabe, H., Yokobe, A., Asai, K., Jackson, B. V., \& Hick, P. L. 1998, J. Geophys. Res., 103, 1981

Kumar, S., \& Broadfoot, A. L. 1979, ApJ, 228, 302

Lallement, R., Bertaux, J. L., \& Kurt, V. G. 1985, J. Geophys. Res., 90, 1413

Lallement, R., Holzer, T. E., \& Munro, R. H. 1986, J. Geophys. Res., 91, 6751

Lamy, P., Llebaria, A., \& Quemerais, E. 2002, Adv. Space Res., 29, 373

Lamy, P. L., Quemerais, E., Llebaria, A., Bout, M., Howard, R., Schwenn, R. \& Simnett, G. 1997, in 5th SOHO Worskshop, The Corona and Solar Wind Near Minimum Activity, ed. A. Wilson (ESA SP-404; Noordwijk: ESA), 491
Llebaria, A., Lamy, P. L., \& Koutchmy, S. 1999, in 8th SOHO Workshop, Plasma Dynamic and Diagnostics in the Solar Transition Region and Corona, ed. J.-C. Vial \& B. Kaldeich-Schümann (ESA SP-446; Noordwijk: ESA), 441 Munro, R. H., \& Jackson, B. V. 1977, ApJ, 213, 874

Ogawa, H. S., Wu, C. Y. R., Gangopadhyay, P., \& Judge, D. L. 1995, J. Geophys. Res., 100, 3455

Phillips, J. L. et al. 1995, GeoRL, 22, 3301

Quémerais, E., Lallement, R., Ferron, S., Koutroumpa, D., Bertaux, J. L., Kyrölä, E., \& Schmidt, W. 2006, J. Geophys. Res., 111, A09114

Quémerais, E., \& Lamy, P. 2002, A\&A, 393, 295

Raymond, J. C., et al. 1998, ApJ, 508, 410

Rucinski, D., \& Fahr, H. J. 1991, Ann. Geophys., 9, 102

Sheeley, N. R., Jr. 1997, ApJ, 484, 472

Strachan, L., Panasyuk, A. V., Dobrzycka, D., Kohl, J. L., Noci, G., Gibson, S. E., \& Biesecker, D. A. 2000, J. Geophys. Res., 105, 2345

Strachan, L., Suleiman, R., Panasyuk, A. V., Biesecker, D. A., \& Kohl, J. L. 2002, ApJ, 571, 1008

Thomas, G. E., \& Krassa, R. F. 1971, A\&A, 11, 218

Zouganelis, I., Meyer-Vernet, N., Landi, S., Maksimovic, M., \& Pantellini, F. 2005, ApJ, 626, L117 